\title{
Motion Artifact Suppression in Ambulatory ECG with Feed Forward Combined Adaptive Filter
}

\author{
Huanqian Zhang ${ }^{1,3}$, Shulin Zhang ${ }^{1}$, Qinghui Jin $^{1}$, Xuehua Liu $^{2}$, Qing Li², Jian Yang ${ }^{2}$, Jianlong Zhao ${ }^{1}$ \\ ${ }^{1}$ Shanghai Institute of Microsystem and Information Technology, Chinese Academy of Sciences, \\ Shanghai, China \\ ${ }^{2}$ Xuhui District Central Hospital, Shanghai, China \\ ${ }^{3}$ University of Chinese Academy of Sciences, Beijing, China
}

\begin{abstract}
Ambulatory electrocardiogram (AECG) is widely used for heart disease diagnosis and treatment after surgery, but the signal quality will be negatively affected by the motion artifact (MA) coupling in the AECG signal. Adaptive filter (AF) has been used to suppress MA from AECG. Unfortunately, because of the non-stationary property of AECG and MA, the distortion and the artifact suppression performance of the AF's output cannot be satisfied simultaneously, resulting in a poor diagnosis, such as: low QRS beat detection accuracy.

This paper proposes a new kind of AF: Feed Forward Combine AF (FFC-AF). It is composed by two separate $A F$ s (one fast convergence speed AF 'FCS-AF' and one high convergence accuracy $A F$ ( $H C A-A F$ ') and one combination parameter which varies with the estimation of reference signal stationary. The performance of FFC-AF is evaluated by distortion, signal artifact ratio and $Q R S$ beat detection accuracy.
\end{abstract}

\section{Introduction}

The signal quality of ambulatory electrocardiogram (AECG) will be corrupted by the motion artefact (MA). As the frequency of MA is overlapped with AECG signal, the ordinary filter cannot remove MA without distorting AECG signal [1, 2].

Adaptive filter (AF) is widely used in biomedical signal processing [3] because of its robustness and simplicity [4]. It uses a reference signal (RS) which is uncorrelated with the original signal (OS), but correlated with the noise signal. Several reports have indicated that electrodes tissue impedance (ETI) has strong correlation with MA [2, 5]. The adaptive searching algorithm will adjust the filter parameter to obtain AF's output, which is the optimum estimation of the real noise.

In order to suppress MA in AECG, different kinds of AFs have been reported. Variable step size least mean square AF (VSS-LMS-AF) [6] was used to remove MA in the AECG signal. Two cascade AFs [7] were applied to prevent the signal saturation and track large sudden MA quickly. However, significant amount of distortion on the filtered AECG signal was observed from several AFs [5].

As both of the MA and AECG signals have nonstationary properties [8], VSS-LMS-AF has to vary its step size to track MA variations. The lag of tracking will increase the misadjustment of the output signal [9]. Low signal distortion and high signal to artifact ratio (SAR) cannot be satisfied at the same time. More researches are required to find a new approach.

In this paper, we propose a novel AF 'feed forward combine AF (FFC-AF)' for suppressing MA in AECG. We investigate its SAR in MA state (AECG with baseline fluctuations (BF)), its distortion in MA free state (AECG without $\mathrm{BF}$ ) and its QRS beat detection (BD) accuracy in both states.

\section{Method}

\subsection{LMS-AF and VSS-LMS-AF}

Figure. 1. displays the structure of AF used in noise cancelling application [10].

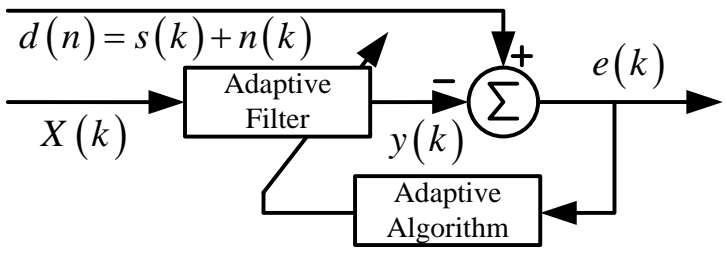

Fig. 1. The block diagram of noise cancelling AF

where $s(k)$ is the OS without noise signal $n(k), X(k)$ is the RS correlated with $n(k), d(k)=s(k)+n(k)$ is the desired signal $(\mathrm{s}(\mathrm{k})$ contaminated by $n(\mathrm{k}))$, $\mathrm{e}(\mathrm{k})$ is the error signal. The filter output is $\mathrm{y}(\mathrm{k})=\mathrm{W}^{\mathrm{T}}(\mathrm{k}) \cdot \mathrm{X}(\mathrm{k})$ and the transposition of filter parameters is $\mathrm{W}^{\mathrm{T}}(\mathrm{k})$ named as weight vector. $\mathrm{k}$ is the iteration number. The difference equation of filter parameters is given by (1):

$\mathrm{W}(\mathrm{k}+1)=\mathrm{W}(\mathrm{k})+\mu \cdot \mathrm{e}(\mathrm{k}) \cdot \mathrm{X}(\mathrm{k})$

where $\mu$ is the step size of LMS-AF. For VSS-LMS-AF, 
$\mu(\mathrm{k})$ replaces $\mu$ and varies with iteration number $\mathrm{k}$.

When the LMS-AF and VSS-LMS-AF have converged, the filter output $y(\mathrm{k})$ is the best estimation of the real noise $n(k)$. By subtracting the estimated noise signal $y(k)$ from desired signal $d(k)=s(k)+n(k)$, we can obtain the optimum estimation of the $s(k)(2)[4]$ :

$$
\mathrm{E}\left[\mathrm{e}^{2}(\mathrm{k})\right]=\mathrm{E}\left\{[\mathrm{d}(\mathrm{k})-\mathrm{y}(\mathrm{k})]^{2}\right\}=\mathrm{E}\{[\mathrm{s}(\mathrm{k})+\mathrm{n}(\mathrm{k})-
$$$$
\left.\mathrm{y}(\mathrm{k})]^{2}\right\}=\mathrm{E}\left[\mathrm{s}^{2}(\mathrm{k})\right]
$$

\subsection{Misadjustment of LMS AFs}

In the equation (2), as $y(k)$ is the estimation of $n(k)$, it contains estimation misadjustment which leads to the distortion of the filter's output.

As both AECG and MA have non-stationary properties, this will force the AF out of convergence state [11]. In nonconvergence state, AF algorithm has to search the optimum solution of its weight vectors. The search lag will increase misadjustment and lead to output distortions of AF. The overall misadjustment of AF can be shown [9]:

$$
\mathrm{M}_{\text {total }}=\mathrm{M}_{\text {excess }}+\mathrm{M}_{\text {lag }} \approx \frac{\mu \cdot \operatorname{tr}[\mathrm{R}]}{2}+\frac{\operatorname{tr}[\mathrm{Q}]}{2 \cdot \mu \cdot \delta_{\mathrm{v}}^{2}}
$$

where $M_{\text {excess }}$ is the misadjustment from convergence accuracy in stationary state and $\mathrm{M}_{\text {lag }}$ is the misadjustment from tracking lag in non-stationary state. $\operatorname{tr}[\mathrm{R}]$ and $\operatorname{tr}[\mathrm{Q}]$ are the trace of the autocorrelation matrix of RS and the trace of the autocorrelation matrix of desire signal, $\delta_{\mathrm{v}}^{2}$ is the variance of $\mathrm{n}(\mathrm{k})$ in the desire signal.

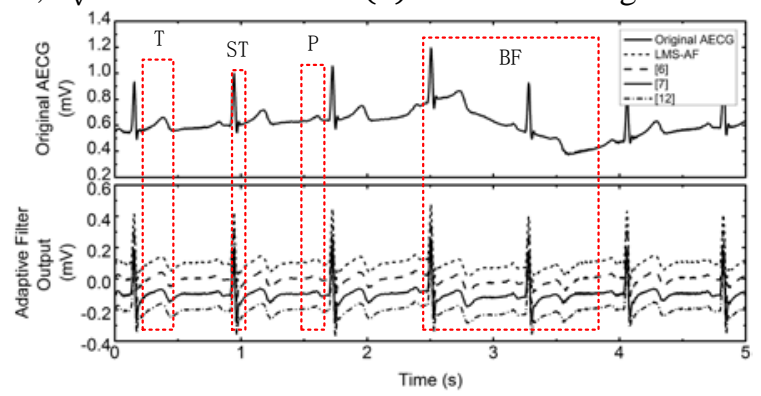

Fig. 2. Output of LMS-AF and VSS-LMS-AFs

We can see the output of different LMS-AF and VSSLMS-AFs [6, 7, 12] in Fig 2. Compared with the original AECG signal, there are obvious AF output distortions as indicated by the dashed boxes.

1. T and P wave, ST segment distortions are in MA free state. The non-stationary AECG signal increases $\mathrm{M}_{\text {excess }}$ and leads to these distortions.

2. BF distortions are in MA state. The non-stationary MA signal increases $\mathrm{M}_{\text {lag }}$ and leads to distortions.

In order to reduce distortions, we should increase convergence accuracy in MA free state to decrease $M_{\text {excess }}$ introduced by the non-stationary AECG signal; and increase convergence speed in MA state to decrease $\mathrm{M}_{\text {lag }}$ introduced by the non-stationary MA signal.

\subsection{Feed forward combine AF}

We propose a new kind of AF 'feed forward combine AF (FFC-AF)' for suppressing MA in AECG. Fig. 3 describes its structure and (4) shows its equation.

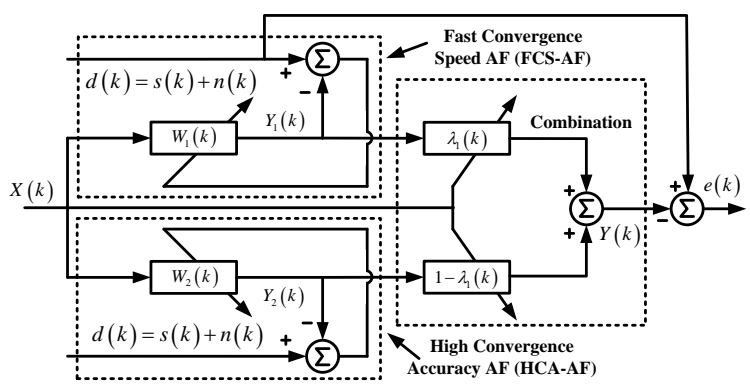

Fig. 3 FFC-AF block diagram

$$
\left\{\begin{array}{c}
\mathrm{Y}(\mathrm{k})=\lambda_{1}(\mathrm{k}) \cdot \mathrm{Y}_{1}(\mathrm{k})+\left[1-\lambda_{1}(\mathrm{k})\right] \cdot \mathrm{Y}_{2}(\mathrm{k}) \\
\mathrm{Y}_{1,2}=\mathrm{W}_{1,2}^{\mathrm{T}}(\mathrm{k}) \cdot \mathrm{X}(\mathrm{k}) \\
\lambda_{1}(\mathrm{k})=\frac{2}{1+\exp [-\mathrm{p} \cdot \delta(\mathrm{k})]}-1 \\
\delta(\mathrm{k})=\frac{1}{\mathrm{n}} \sum_{\mathrm{i}=0}^{\mathrm{n}-1}\left(\mathrm{x}_{\mathrm{i}}-\frac{1}{\mathrm{n}} \sum_{\mathrm{i}=0}^{\mathrm{n}-1} \mathrm{x}_{\mathrm{i}}\right)^{2} \\
\mathrm{p}= \begin{cases}\mathrm{p}_{\text {non }} & \delta(\mathrm{k}) \geq \delta_{\text {th }} \\
\mathrm{p}_{\text {sta }} & \delta(\mathrm{k})<\delta_{\text {th }}\end{cases}
\end{array}\right.
$$

where $\mathrm{X}(\mathrm{k})$ is the RS of two separate filters. Parameters $\lambda_{1}(\mathrm{k})$ is the combination weight of the filter output, which lies between 0 and 1 . At each iteration, $\lambda_{1}(\mathrm{k})$ will update its value according to the stationary degree of $\mathrm{X}(\mathrm{k})$ by its variance $\delta(\mathrm{k})$ [7]. When the $\mathrm{X}(\mathrm{k})$ is in stationary state, $\delta(\mathrm{k})$ is lower than the threshold $\delta_{\text {th }}$ and this means that AF is in MA free state. Then $\lambda_{1}(\mathrm{k})$ will maintain around 0 to increase the weight of high convergence accuracy AF (HCA-AF) output. When RS is in non-stationary state, $\delta(\mathrm{k})$ is larger than $\delta_{\text {th }}$ and this means that AF is in MA state. Then $\lambda_{1}(\mathrm{k})$ will maintain around 1 to increase the weight of fast convergence speed AF (FCS-AF) output.

The feed forward path enables a fast response speed from RS to combination parameter $\lambda_{1}(\mathrm{k})$. So $\lambda_{1}(\mathrm{k})$ has a short transition delay between MA state and MA free state to ensure small distortions.

Moreover, in MA free state, the feed forward path can bypass the variation of the error signal $\mathrm{e}(\mathrm{k})$. Parameter $\lambda_{1}(\mathrm{k})$ will maintain above 0 slightly, so that HCA-AF has a high weight to ensure small distortions.

$\mathrm{Y}_{1,2}(\mathrm{k})$ are the outputs of two separate filters at time $\mathrm{k}$ and $\mathrm{W}_{1,2}^{\mathrm{T}}(\mathrm{k})$ are the weight vectors. We use two different algorithms: normalized LMS (NLMS) and variable step size NLMS (VSS-NLMS) to update step sizes:

$$
\left\{\begin{array}{c}
\mu_{1}=\beta_{1} / \mathrm{X}^{\mathrm{T}}(\mathrm{k}) \cdot \mathrm{X}(\mathrm{k}) \\
\mu_{2}(\mathrm{k}+1)=\alpha_{2} \cdot \mu_{2}(\mathrm{k})+\beta_{2} / \mathrm{X}^{\mathrm{T}}(\mathrm{k}) \cdot \mathrm{X}(\mathrm{k})
\end{array}\right.
$$

where $\beta_{1}, \alpha_{2}, \beta_{2}$ are parameters to control the step size. Because the NLMS algorithm has good convergence stability and fast convergence speed, it is used in FCS-AF. Because VSS-NLMS algorithm can smooth the fluctuation of $\mathrm{X}(\mathrm{k})$, it is used in HCA-AF. 
We used the AECG and RS from our previous research [13] and preprocessed by Labview. The AECG and the RS were filtered by a $0.5 \mathrm{~Hz}$ to $100 \mathrm{~Hz}$ band-pass filter following with a band-stop filter with $50 \mathrm{~Hz}$ notch frequency. The $\mathrm{p}_{\text {non }}, \mathrm{p}_{\text {sta }}, \mathrm{n}$ and $\delta_{\text {th }}$ in (4) were 1,80 , 1500 and 0.0115 . The step size parameters in (5) were $\beta_{1}=0.02, \alpha_{2}=0.05$ and $\beta_{2}=0.0009$. The length of filter was 200 taps.

\subsection{Distortion}

AF output distortion can be measured by correlation between the filter output and the original AECG. The overall 705 MA free cardiac cycles (CCs) were selected manually from 1143 CCs. Fig. 4 (Cor: Correlation) shows one example of MA free CC.

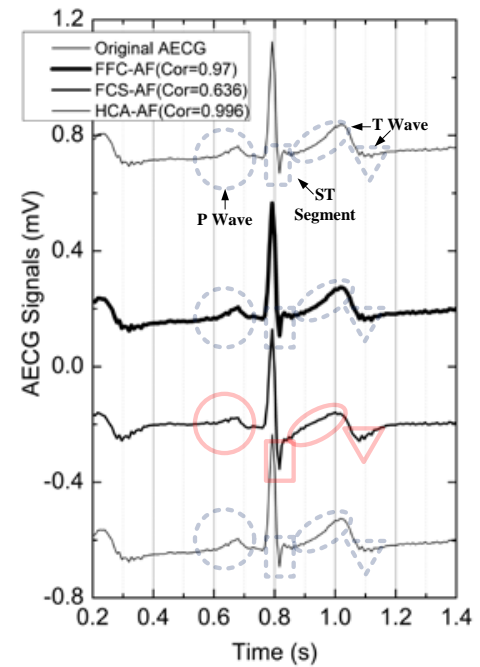

Fig. 4 MA free CC and its AFs' output

We can see that the $\mathrm{P}$ and $\mathrm{T}$ wave and ST segment of FCS-AF have great distortion with the original AECG, but these segments of FFC-AF and HCA-AF have no obvious distortion. Table 1 (STD: Standard deviation) is the statistical result of the correlation analysis. The median value of ten experiments data shows that the output distortion of FFC-AF ( $\operatorname{Cor}_{\mathrm{FFC}-\mathrm{AF}}=0.873$ ) is close to HCA-AF $\left(\operatorname{Cor}_{\mathrm{HCA}-\mathrm{AF}}=0.901\right)$ and much stronger than FCS-AF $\left(\right.$ Cor $\left._{\mathrm{FCS}-\mathrm{AF}}=0.352\right)$.

Table 1. Cor between AF output and OS

\begin{tabular}{cccc}
\hline \hline Cor & FFC-AF & FCS-AF & HCA-AF \\
\hline Median & 0.873 & 0.352 & 0.901 \\
STD & 0.02 & 0.02 & 0.03 \\
\hline \hline
\end{tabular}

That is, FFC-AF has almost the lowest distortion.

\subsection{Signal to artifact ratio}

We use SAR in (6) as an index, to visualize MA suppression performance (SP) of FFC-AF, FCS-AF and HCA-AF. A higher SAR indicates a better MA SP.

$$
\mathrm{SAR}=\delta_{\mathrm{ecg}}^{2} /\left(\delta_{\text {ecg_MA }}^{2}-\delta_{\text {ecg }}^{2}\right)=\delta_{\text {ecg }}^{2} / \delta_{\mathrm{MA}}^{2}
$$

where $\delta_{\text {ecg }}^{2}$ is the variance of MA free AECG signal which is extracted by the first three seconds of MA free AECG signal (three or four CCs) and $\delta_{\mathrm{MA}}^{2}$ is the variance of the MA which is calculated by subtracting $\delta_{\text {ecg }}^{2}$ from the variance ( $\delta_{\text {ecg_MA }}^{2}$ ) of the MA segments (six segments in one experiment) from the same experiment.

Table 2. SAR of different original AECG and AF

\begin{tabular}{ccccc}
\hline \hline \multirow{2}{*}{ SAR } & $\begin{array}{c}\text { AECG } \\
\text { with MA }\end{array}$ & $\begin{array}{c}\text { FFC } \\
\text { AF }\end{array}$ & $\begin{array}{c}\text { FCS } \\
\text { AF }\end{array}$ & $\begin{array}{c}\text { HCA } \\
\text { AF }\end{array}$ \\
\hline Med & -15.59 & 1.72 & 15.76 & -14.42 \\
STD & 5.45 & 7.38 & 12.37 & 5.54 \\
\hline \hline
\end{tabular}

Table 2 (Med: Median) shows the SAR from ten experiments statistically. It is observed that FFC-AF has moderate, FCS-AF has the highest and HCA-AF has the lowest SAR. That is, FFC-AF sacrifices MA SP.

\subsection{QRS beat detection}

We employed the QRS BD algorithm 'wqrs' [14] from PhysioNet [15] to evaluate the diagnosis performance on the AF corrected AECG signal. Fig 5 shows the result. One triangle represents one QRS beat detected by wqrs.

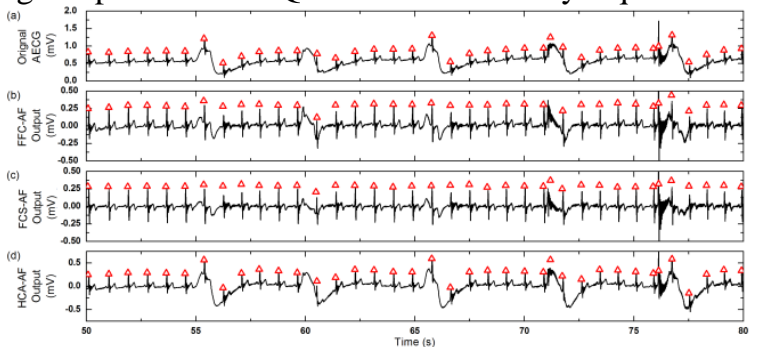

Fig. 5 QRS BD performance analysis

We analyzed the overall 1143 CCs from ten experiments (MA CCs and MA free CCs were all included). Performance of the QRS BD is measured in terms of Positive Predictivity ( $+\mathrm{P}$ : the number of true $\mathrm{BD}$ in all $\mathrm{BD})$ and the Sensitivity (Se: the number of true BD in all beats), which are calculated as:

$$
+\mathrm{P}=\frac{\mathrm{TP}}{\mathrm{TP}+\mathrm{FP}}, \mathrm{Se}=\frac{\mathrm{TP}}{\mathrm{TP}+\mathrm{FN}}
$$

where TP is the true positive, FN is the false negative and FP is the false positive QRS BD [16].

Table 3. $+\mathrm{P}$ and Se of original AECG and AF corrected AECG signal

\begin{tabular}{ccccc}
\hline \hline$+\mathbf{P}$ & Orig & FFC & FCS & HCA \\
$(\%)$ & AECG & AF & AF & AF \\
\hline M & 99.09 & 99.71 & 99.62 & 99.09 \\
STD & 1.66 & 0.47 & 0.66 & 1.66 \\
\hline \hline Se & Orig & FFC & FCS & HCA \\
$(\%)$ & AECG & AF & AF & AF \\
\hline M & 99.28 & 99.64 & 99.54 & 99.28 \\
STD & 1.33 & 0.87 & 0.88 & 1.33 \\
\hline \hline
\end{tabular}

Table 3 (Orig: Original, M: Mean) shows that FFC-AF $\left(+\mathrm{P}_{\mathrm{FFC}-\mathrm{AF}}=99.71 \%\right.$ and $\left.\mathrm{Se}_{\mathrm{FFC}-\mathrm{AF}}=99.64 \%\right)$ has better performance than HCA-AF $\left(+\mathrm{P}_{\mathrm{HCA}-\mathrm{AF}}=99.09 \%\right.$ 
and $\left.\mathrm{Se}_{\mathrm{HCA}-\mathrm{AF}}=99.28 \%\right)$, FCS-AF $\quad\left(+\mathrm{P}_{\mathrm{FCS}-\mathrm{AF}}=\right.$ $99.62 \%$ and $\left.\mathrm{Se}_{\mathrm{HCA}-\mathrm{AF}}=99.54 \%\right)$ and original AECG $\left(+\mathrm{P}_{\text {Orig }}=99.09 \%\right.$ and $\left.\mathrm{Se}_{\text {Orig }}=99.28 \%\right)$. From Table 3, we can see that FFC-AF has the best QRS BD accuracy. It can result in better diagnostic performance.

\section{Discussion}

This paper investigated a new kind of AF 'FFC-AF' for suppressing MA in AECG. FFC-AF is formed by two separate AFs: FCS-AF and HCA-AF. All of the three AFs' performances are evaluated by means of distortion (correlation), SAR and QRS BD.

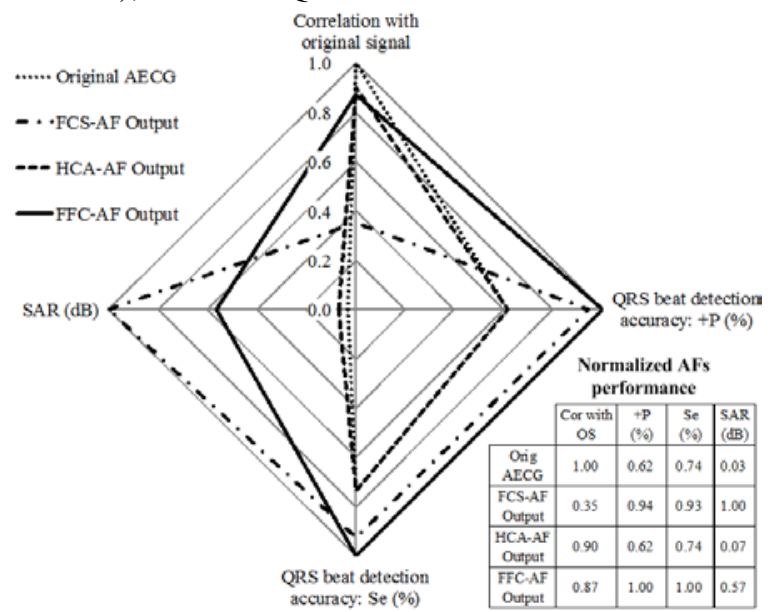

Fig 6. Radar plot of normalized AFs performance

Figure 6 summarized the normalized performance of three AFs. HCA-AF had almost the same $+\mathrm{P}$, Se and SAR as the OS, as it had the strongest correlation with the OS. In all AFs, FFC-AF had almost the lowest distortion, moderate SAR and the highest QRS BD accuracy.

Because of the fast response of feed forward path from RS to combination parameter and its bypass of error signal, FFC-AF slightly sacrifices MA SP for a great reduction of distortion.

Thus FFC-AF could suppress MA in AECG and maintained low output distortion. FFC-AF can be used to study other characteristics of AECG signal, such as P and $\mathrm{T}$ waves or QRS morphology.

\section{Acknowledgements}

This work was supported by grants from Science and Technology Commission of Shanghai Municipality (Grant No. 14521106200). The authors wish to express their sincere gratitude to Prof. Zarzoso, from the University of Nice Sophia Antipolis, for his kind guidance with the AF algorithm and his feedback on an early version of the manuscript. The authors appreciate Dr. Teo and Dr. Feng of IHPC for their careful review of the manuscript.
[1] N. V. Thakor, et al., "Estimation of QRS complex power spectra for design of a QRS filter," IEEE Trans. Biomed. Eng, vol. 31, pp. 702-706, 1984.

[2] D. Buxi, et al., "Correlation Between Electrode-Tissue Impedance and Motion Artifact in Biopotential Recordings," Sensors Journal, IEEE, vol. 12, pp. 3373-3383, 2012.

[3] V. K. Iyer, et al., "Adaptive filtering in biological signalprocessing," Critical Reviews in Biomedical Engineering, vol. 17, pp. 531-584, 1990.

[4] B. Widrow, et al., "Adaptive noise cancelling: Principles and applications," Proceedings of the IEEE, vol. 63, pp. 16921716, 1975.

[5] I. Romero, et al., "Adaptive filtering in ECG denoising: A comparative study," Computing in Cardiology 2012:39:4548.

[6] P. S. Hamilton, et al., "Comparison of methods for adaptive removal of motion artifact," Computers in Cardiology 2000;27:383-386.

[7] K. Hyejung, et al., "Motion artifact removal using cascade adaptive filtering for ambulatory ECG monitoring system," in Biomedical Circuits and Systems Conference (BioCAS) IEEE, 2012, pp. 160-163.

[8] G. D. Clifford, "ECG statistics, noise, artifacts, and missing data," Advanced Methods and Tools for ECG Data Analysis, pp. 55-99, 2006.

[9] S. Haykin, Adaptive filter theory (3rd ed.): Prentice-Hall, Inc., 1996.

[10] P. S. Diniz, Adaptive Filtering: Algorithms and Practical Implementation: Kluwer Academic Publishers, 2002.

[11] B. Widrow, et al., "Stationary and nonstationary learning characteristics of the LMS adaptive filter," Proceedings of the IEEE, vol. 64, pp. 1151-1162, 1976.

[12] L. Wen-Ching, et al., "Adaptive reduction of motion artifact in a portable ECG system," in Sensors,, 2010, pp. 704-707.

[13] H. Zhang, et al., "A multi-channel electrode tissue impedance detection approach for motion artifact suppression in ambulatory electrocardiography," Computing in Cardiology 2015;42:117-120.

[14] W. Zong, et al., "A robust open-source algorithm to detect onset and duration of QRS complexes," Computers in Cardiology 2003;30:737-740.

[15] A. L. Goldberger, et al., "Physiobank, physiotoolkit, and physionet components of a new research resource for complex physiologic signals," Circulation, vol. 101, pp. e215-e220, 2000.

[16] R. Parikh, et al., "Understanding and using sensitivity, specificity and predictive values," Indian Journal of Ophthalmology, vol. 56, pp. 45-50, 2008.

Address for correspondence.

Jianlong Zhao

865 ChangNing Road, Shanghai, China

jlzhao@mail.sim.ac.cn

\section{References}

